\title{
FUNGOS INGOLDIANOS ASSOCIADOS A FOLHAS SUBMERSAS DE RIACHOS EM DIFERENTES BIOMAS DO BRASIL
}

\author{
Mell Caroline Oliveira Amorim¹; Patrícia Oliveira Fiuza²; Luís Fernando \\ Pascholati Gusmão ${ }^{3}$ \\ 1.Bolsista PIBIC/CNPq, Graduanda em Bacharelado em Ciências Biológicas, Universidade Estadual de Feira de \\ Santana, e-mail: mellc.oliveira@gmail.com \\ 2. Bolsista Pós-Doutorado Júnior/CNPq, Universidade Estadual de Feira de Santana, e-mail: \\ patyfiuzabio@gmail.com \\ 3. Orientador, Departamento de Ciências Biológicas, Universidade Estadual de Feira de Santana, e-mail: \\ lgusmao.uefs@gmail.com
}

PALAVRAS-CHAVE: Ecossistemas aquáticos, hifomicetos aquáticos, tropical.

\section{INTRODUÇÃO}

$\mathrm{O}$ grupo dos fungos ingoldianos é constituído por fungos que correspondem à fase assexual de Ascomycota ou Basidiomycota, esporulam em ambiente aquático e ocorrem nas folhas em decomposição de córregos e rios (Bärlocher, 1992; Duarte et al., 2015). Os fungos ingoldianos apresentam conídios com estrutura tetrarradiada, multirradiada ou sigmóide, que é o principal caráter morfológico utilizado para a taxonomia (Bärlocher 1992; Bashien et al. 2013). Estes fungos detêm grande importância devido a sua eficiência na ciclagem de nutrientes dos substratos presentes no ambiente aquático (Gareth Jones \& Pang, 2012). Estudos com fungos ingoldianos são realizados no Brasil desde o final dos anos 80, mas ainda são bastante pontuais e escassos Fiuza et al., 2015; Fiuza \& Gusmão, 2017).

\section{METODOLOGIA}

O material estudado foi previamente coletado e processado por membros do projeto Aquaripária no ano de 2012. Posteriormente as amostras de suspensão de esporos foram encaminhadas ao Laboratório de Micologia da Universidade Estadual de Feira de Santana (LAMIC/UEFS) onde foram desenvolvidas as atividades propostas no plano de trabalho. A suspensão de esporos passou pelo processo de filtração em filtros de miliporo $(0,5 \mu \mathrm{m}$ de poro) (Figura 1A-B) e posteriormente os filtros foram corados com azul de algodão (Figura 1C), montados em lâminas e selados com esmalte incolor (Figura 1C). Após montagem das lâminas o material foi examinado em microscópio (Figura 1D) e devidamente identificado com base na literatura.

\section{RESULTADOS E DISCUSSÃO}

Foi examinado um total de 122 lâminas de amostras de suspensão de esporos das quatro localidades citadas. Desse total, 43 lâminas são de Salvador, 15 de Palotina, 43 de Erechim e 21 de Brasília.No presente estudo foram observados cinco gêneros de fungos ingoldianos nas lâminas observadas - Campylospora, Mycofalcella, Lunulospora, Trinacrium e Anguillospora, em duas das quatro localidades (Erechim e Palotina) (Tabela 1).

Tabela 1: Gêneros de fungos ingoldianos registrados nos riachos das áreas de estudo. 


\begin{tabular}{lcccc}
\hline \multicolumn{1}{c}{ Espécie } & Erechim & Salvador & Brasília & Palotina \\
\hline $\begin{array}{l}\text { Anguillospora } \\
\text { Ingold }\end{array}$ & $\mathrm{X}$ & - & - & $\mathrm{X}$ \\
$\begin{array}{l}\text { Campylospora } \\
\text { Ranzoni }\end{array}$ & $\mathrm{X}$ & - & - & $\mathrm{X}$ \\
$\begin{array}{l}\text { Heliscus Sacc. } \\
\begin{array}{l}\text { Lunulospora } \\
\text { Ingold }\end{array}\end{array}$ & - & - & - & \\
$\begin{array}{l}\text { Mycofalcella } \\
\text { Marvanová, Om- }\end{array}$ & $\mathrm{X}$ & - & - & $\mathrm{X}$ \\
Kalth. \& J. \\
Webster
\end{tabular}

Em Palotina foram encontrados seis gêneros de fungos ingoldianos (Tabela 1). Os gêneros foram encontrados em 11 das 15 lâminas observadas. Estes gêneros, com exceção de Heliscus, ocorrem geralmente em região tropical com alta frequência (Fiuza et al. 2017, Betancourt-López \& Santos-Flores 1997). Heliscus só ocorreu nas amostras de Palotina (Figura 2). Em Erechim foram encontrados cinco gêneros comuns aos de Palotina, todos comuns a Palotina (Tabela 1, Figura 2). Os resultados se assemelham aos encontrados por Schoenlein-Crusius et al. (2015) que relataram a ocorrência de gêneros similares de fungos ingoldianos no Parque da Aclimatação (SP), que também consiste em área de Mata Atlântica. Nas amostras oriundas de Salvador e Brasília (DF), não foram observados fungos ingoldianos (Figura 2). Fiuza et. al (2017) em estudo da diversidade de fungos ingoldianos no Brasil registrou 53 novos táxons para a Mata a Atlântica e 21 para o Cerrado, além de 19 novos registros para a Amazônia e 39 para a Caatinga. Em vista disso, vê-se a necessidade de ampliação dos estudos sobre fungos ingoldianos no Brasil, para conhecer a diversidade deste grupo de fungos.

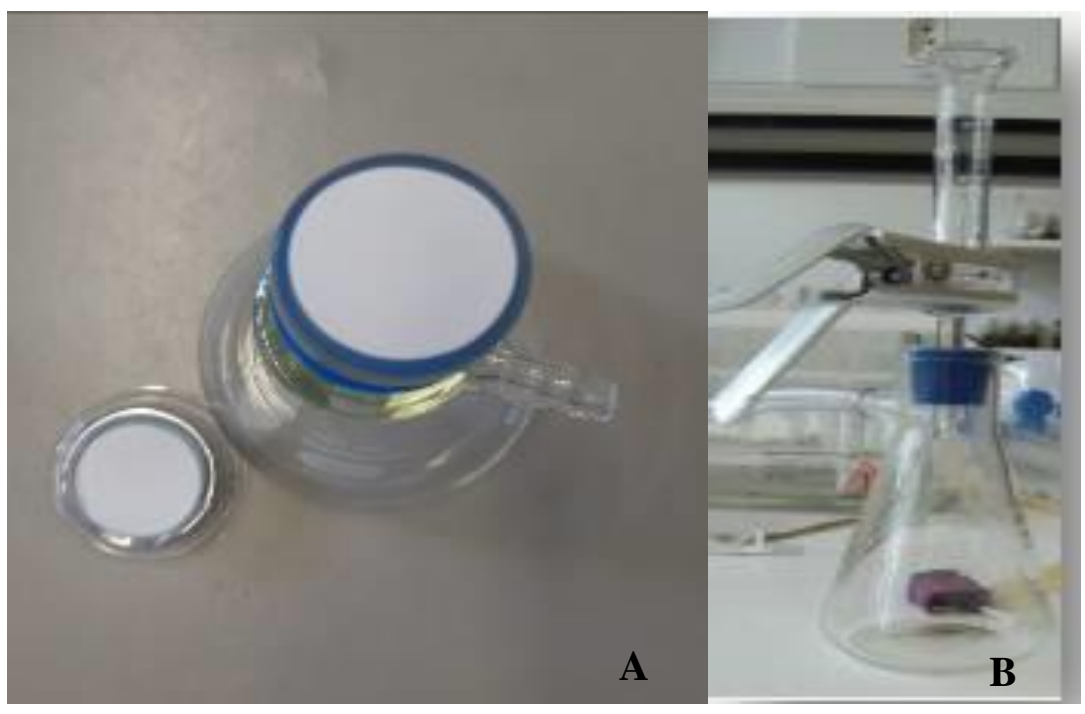




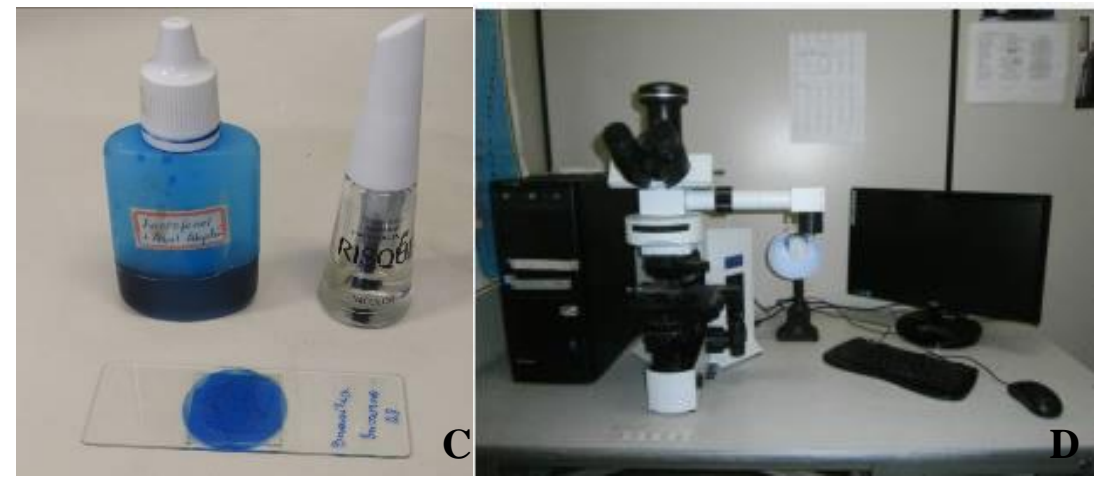

Figura 1: A-B - Vidraria para filtração do material e filtro de miliporo; C-Lâmina confeccionada com azul de algodão e selada com esmalte incolor; D- Identificação e contagem de conídios em microscópio.

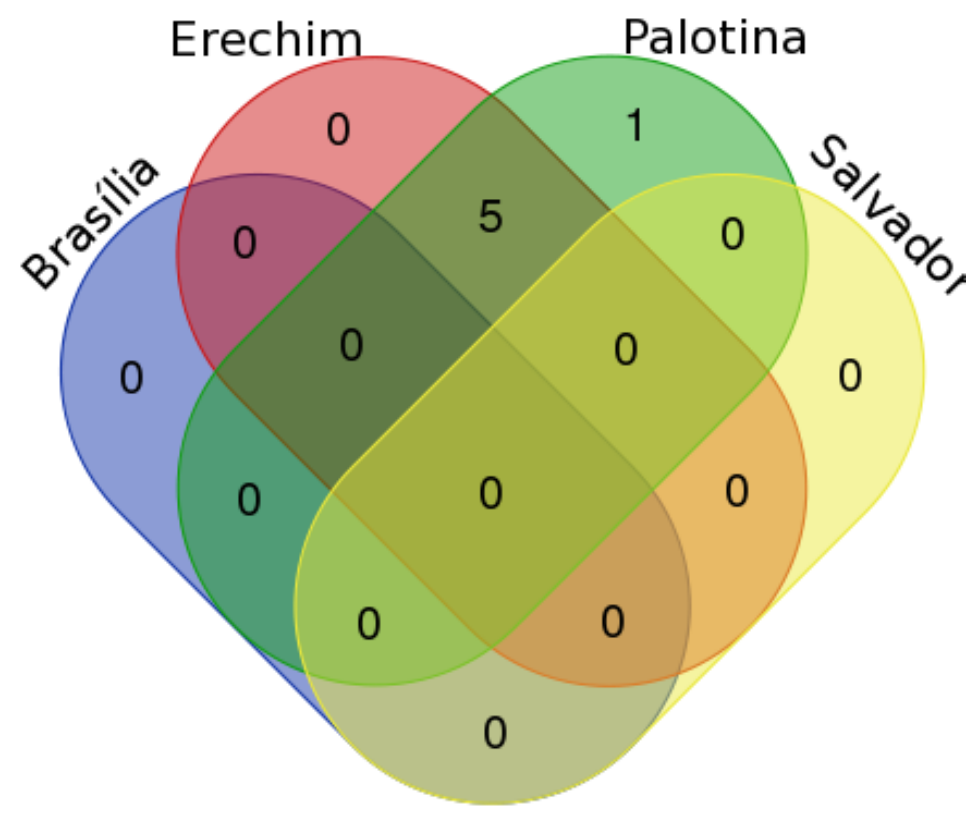

Figura 2: Diagrama de Venn que mostra o número de gêneros de fungos ingoldianos presentes nas diferentes localidades estudadas.

\section{CONCLUSÃO}

Após a observação das lâminas foram encontrados cinco gêneros recorrentes em regiões de clima tropical. No entanto, para as cidades de Erechim e Palotina, estes gêneros constituem novos registros, uma vez que não há estudos sobre fungos ingoldianos nessas áreas. As amostras de Salvador e Brasília não apresentaram nenhum conídio de fungos ingoldianos. Os gêneros foram encontrados nas regiões de bioma Mata Atlântica, com exceção de Salvador, que não foi observada nenhuma ocorrência, onde também não existem estudos sobre estes fungos. Tendo em vista a escassez de estudos com fungos ingoldianos é nítida a necessidade de ampliação do conhecimento destes fungos no Brasil. 


\section{REFERÊNCIAS}

BASCHIEN, C.; TSUI, C. KM.; GULIS, V.; SZEWZYK, U.; MARVANOVA, L. The molecular phylogeny of aquatic hyphomycetes with affinity to the Leotiomycetes. Fungal biology. p.: 660-672. 2013.

BÄRLOCHER, F. Research on aquatic hyphomycetes: historical background and overview. In: BARLOCHER, F. (ed.) Ecology of aquatic hyphomycetes. Ecological Studies. Berlin: Spring-Verlag. p.: 1-15. 1992.

BETANCOURT-LÓPEZ, C. SANTOS-FLORES, C. J.; Aquatic and water-borne Hyphomycetes (Deuteromycotina): in streams of Puerto Rico, including records from other Neotropical locations. College of Arts and Sciences, University of Puerto Rico, 1997.

BORLAUG, N.E. Feeding a world of 10 billion people: the miracle ahead. In: R. Bailey (ed.). Global warming and other eco-myths. pp. 29-60. Competitive Enterprise Institute, Roseville, EUA. 2002.

DUARTE, S.; BAERLOCHER, F; PASCOAL, C; CASSÍO, F. Biogeography of aquatic hyphomycetes: Current knowledge and future perspectives. Fungal Ecology. 1-10. 2015.

FIUZA, P.O.; GUSMÃO, L.F.P. Ingoldian fungi from the semi-arid Caatinga biome of Brazil. Mycosphere 4 (6): 1133-1150. 2013.

FIUZA, P. O., DE PAIVA OTTONI-BOLDRINI, B. M., MONTEIRO, J. S., CATENA, N. R., HAMADA, N., \& GUSMÃO, L. F. P. First records of Ingoldian fungi from the Brazilian Amazon. Brazilian Journal of Botany, 38(3), 615-621. 2015.

FIUZA, PATRÍCIA O. et al. Ingoldian fungi of Brazil: some new records and a review including a checklist and a key. Phytotaxa, v. 306, n. 3, p. 171-200. 2017.

FUNDAÇÃO SOS MATA ATLÂNTICA, INPE \& INSTITUTO SOCIOAMBIENTAL. Atlas da evolução dos remanescentes florestais da Mata Atlântica e ecossistemas associados no período de 1990-1995. São Paulo. 1998.

FUNDAÇÃO SOS MATA ATLÂNTICA \& INPE. Atlas dos remanescentes florestais da Mata Atlântica e ecossistemas associados no período de 1995-2000. Relatório final. São Paulo. 2002.

KLINK, Carlos A.; MACHADO, Ricardo B. A conservação do Cerrado brasileiro. Megadiversidade, v. 1, n. 1, p. 147-155, 2005.

SCHOENLEIN-CRUSIUS, Iracema Helena et al. Richness of ingoldian and facultative aquatic fungi in Parque Municipal do Ibirapuera, São Paulo, Brazil. Hoehnea, v. 41, n. 1, p. 61-76, 2014.

SCHOENLEIN-CRUSIUS, I. H.; MOREIRA, C. G.; GOMES, E. P. C. Riqueza dos fungos ingoldianos e dos fungos aquáticos facultativos do Parque Municipal da Aclimação, São Paulo, SP, Brasil. Hoehnea, v. 42, p. 239-251, 2015.

SCHOENLEIN-CRUSIUS, I. H. et al. Diversity of conidial fungi and some abiotic variables of the water after the reopening of the Pirarungaua stream in the Jardim Botânico, São Paulo, São Paulo State, Brazil. Hoehnea, v. 43, n. 1, p. 57-75, 2016. 